\title{
Septic Arthritis and Osteomyelitis of the Hip by Candida albicans
}

ATUL K. TANEJA, MD, Division of Musculoskeletal Imaging, Diagnostic Center, Hospital do Coração and Teleimagem, Division of Musculoskeletal Radiology, Imaging Department, Hospital Israelita Albert Einstein, São Paulo, Brazil; MARTIN TORRIANI, MD, MSc; F. JOSEPH SIMEONE, MD, Division of Musculoskeletal Imaging and Intervention, Massachusetts General Hospital and Harvard Medical School, Boston, Massachusetts, USA. Address correspondence to Dr. A.K. Taneja, Departamento de Imagem, Hospital Israelita Albert Einstein, Av. Albert Einstein, 627, Morumbi, São Paulo SP, Brazil, CEP 05652-900. E-mail: taneja@einstein.br. J Rheumatol 2014;41:2270; doi:10.3899/jrheum.140354

Predisposing factors such as hemodialysis can lead to septic arthritis by the Candida albicans fungus, and early detection and therapy are essential.

A 68-year-old man with renal disease on hemodialysis was admitted for hip pain with limited range of motion and weight loss for 6 months. Medical history included longstanding Crohn disease treated with steroids. A central venous catheter was removed 6 months earlier and no inflammatory changes were present over the arterial-venous fistula on the left arm. C-reactive protein and erythrocyte sedimentation rate demonstrated progressive elevation, while blood cultures were negative. A positron emission tomography-computed tomography (CT) scan showed intense uptake in the right hip (Figure 1). CT demonstrated cortical erosions at metaepiphyseal region of the femur and acetabulum, associated with capsulo-synovial thickening and iliopsoas bursitis, consistent with septic arthritis and osteomyelitis. Results from a CT-guided joint sampling showed growth of fluconazole-sensitive $C$. albicans and fibroconnective tissue with acute and granulomatous inflammatory changes. As a result of multiple comorbidities and absence of prior antifungal therapy, oral fluconazole therapy was started (400 mg, after dialysis) along with percutaneous catheter-drainage of iliopsoas bursitis. A few months later, the patient died of septic shock from Enterobacter bacteremia and cholangitis.

Septic arthritis by $C$. albicans is uncommon, predomi- nantly arising from hematogenous seeding and occurring in healthy patients or with predisposing factors: broad-spectrum antibiotics, steroids, immunosuppressive therapy, malignancy, rheumatoid arthritis, prosthetic joint, intravenous drug abuse, and hemodialysis ${ }^{1,2,3,4}$. There are no specific clinical or radiologic findings of the causative organism when dealing with fungal arthritis, thus only an appropriate culture will permit the correct diagnosis 5 . Secondary destruction of articular cartilage and progressive bone deformity can occur, therefore early detection and therapy are essential to prevent significant morbidity 4 .

\section{REFERENCES}

1. Kawanabe K, Hayashi H, Miyamoto M, Tamura J, Shimizu M, Nakamura T. Candida septic arthritis of the hip in a young patient without predisposing factors. J Bone Joint Surg Br 2003;85:734-5.

2. Springer J, Chatterjee S. Candida albicans prosthetic shoulder joint infection in a patient with rheumatoid arthritis on multidrug therapy. J Clin Rheumatol 2012;18:52-3.

3. Slenker AK, Keith SW, Horn DL. Two hundred and eleven cases of Candida osteomyelitis: 17 case reports and a review of the literature. Diagn Microbiol Infect Dis 2012;73:89-93.

4. Gamaletsou MN, Kontoyiannis DP, Sipsas NV, Moriyama B, Alexander E, Roilides E, et al. Candida osteomyelitis: analysis of 207 pediatric and adult cases (1970-2011). Clin Infect Dis 2012;55:1338-51.

5. Arias F, Mata-Essayag S, Landaeta ME, Capriles CH, Pérez C, Núñez MJ, et al. Candida albicans osteomyelitis: case report and literature review. Int J Infect Dis 2004;8:307-14.

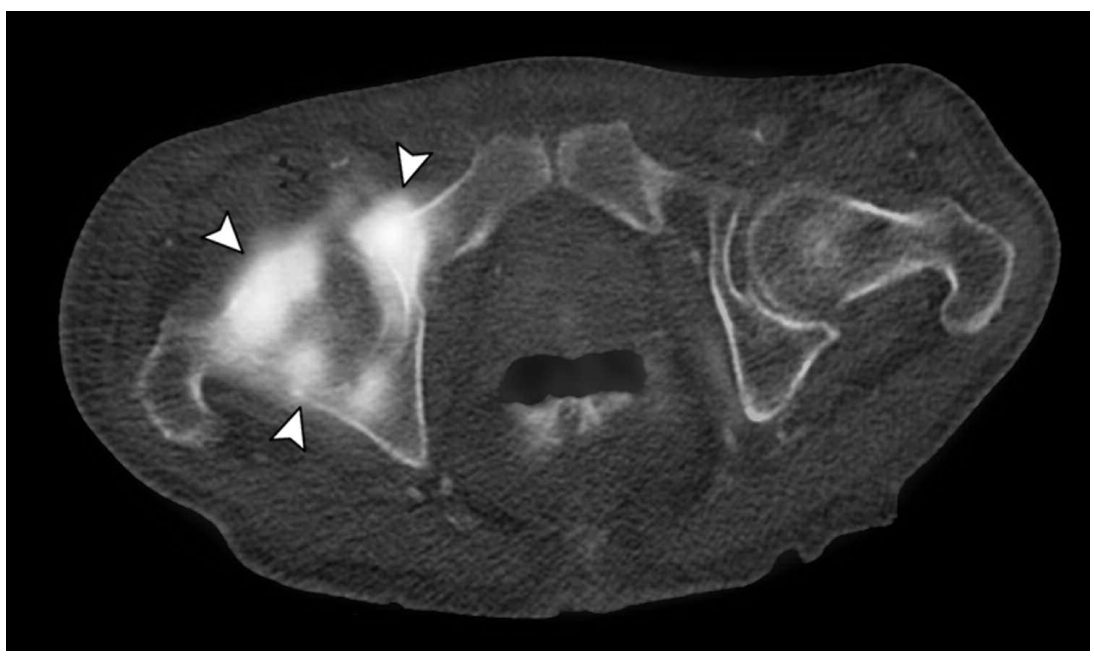

Figure 1. Axial fused PET-CT image of the pelvis shows intense 18-FDG uptake around right hip joint (arrowheads), involving osseous and periarticular soft tissues. PET: positron emission tomography; CT: computed tomography; FDG: fluorodeoxyglucose. 